\title{
Mitogen-activated protein kinase phosphatase-I (MKP-I) impairs the response to anti-epidermal growth factor receptor (EGFR) antibody cetuximab in metastatic colorectal cancer patients
}

\author{
C Montagut ${ }^{*, 1,2}$, M Iglesias 2,3,6,7, M Arumi, 2,3,6,7, B Bellosillo ${ }^{2,3}$, M Gallen ',2, A Martinez-Fernandez', \\ L Martinez-Aviles ${ }^{3}$, I Cañadas ${ }^{1,2}$, A Dalmases ${ }^{1,2}$, E Moragon ${ }^{3}$, L Lema ${ }^{1,2}$, S Serrano ${ }^{2,3,4}$, A Rovira ${ }^{1,2}$, F Rojo ${ }^{2,5}$, \\ J Bellmunt ${ }^{1,2,4,6}$ and J Albanell ${ }^{1,2,4}$ \\ 'Medical Oncology Department, Hospital del Mar-IMAS, Barcelona 08003, Spain; ${ }^{2}$ Cancer Research Program, IMIM-Hospital del Mar, Barcelona

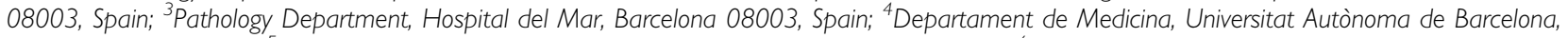 \\ Bellaterra 08193, Spain; ${ }^{5}$ Pathology Department, Fundación Jiménez Díaz, Madrid 28040, Spain; ${ }^{6}$ Department of Medicine, Universitat Pompeu Fabra, \\ Barcelona 08003, Spain
}

\begin{abstract}
BACKGROUND: The validation of KRAS mutations as a negative marker of response to anti-epidermal growth factor receptor (EGFR) antibodies has meant a seminal advance towards treatment individualisation of colorectal cancer (CRC) patients. However, as a KRAS wild-type status does not guarantee a response to anti-EGFR antibodies, a current challenge is the identification of other biomarkers of response. On the basis of pre-clinical evidence, we hypothesised that mitogen-activated protein kinase phosphatase-I (MKP-I), a phosphatase that inactivates MAPKs, could be a mediator of resistance to anti-EGFR antibodies.

METHODS: Tumour specimens from 48 metastatic CRC patients treated with cetuximab-based chemotherapy were evaluated for KRAS and BRAF mutational status and MKP-I expression as assessed by immunohistochemistry.

RESULTS: As expected, clinical benefit was confined to wild-type KRAS and BRAF patients. Mitogen-activated protein kinase phosphatase-I was overexpressed in 16 patients (33\%) and was not associated with patient baseline clinicopathological characteristics and KRAS mutational status. All patients with BRAF mutations $(n=3)$ had MKP-I overexpression. Among KRAS wildtype patients, MKP-I overexpressors had a 7\% response rate (RR), whereas patients not overexpressing MKP-I had a 44\% RR $(P=0.03)$. Moreover, median time to progression was significantly longer in MKP-I non-overexpressing patients (32 vs I 3 weeks, $P=0.009)$.

CONCLUSION: These results support the concept of MKP-I as a promising negative marker of response to cetuximab-based treatment in CRC patients with wild-type KRAS.
\end{abstract}

British Journal of Cancer (2010) I 02, II37-I|44. doi:10.1038/sj.bjc.66056I2 www.bjcancer.com

Published online 16 March 2010

(c) 2010 Cancer Research UK

Keywords: MKP-I; cetuximab; CRC; RAS; molecular marker

Epidermal growth factor receptor (EGFR) is a transmembrane tyrosine kinase receptor that, on ligand binding to its extracellular domain, is activated and autophosphorylated. This activates several intracellular signalling pathways that regulate crucial oncogenic properties, mainly the PI3K-PTEN-AKT and the mitogen-activated protein kinase (MAPK) RAS-RAF-MEKERK cascade (Hanahan and Weinberg, 2000; Albanell et al, 2001; Mendelsohn and Baselga, 2006). Moreover, EGFR inhibits the activation of the other two MAPKs, namely, p38 MAPK and c-Jun NH2-terminal kinase (JNK), which, contrary to ERK, drive apoptotic signals (Dhillon et al, 2007; Takeuchi and Ito, 2010). The unique position of EGFR as a regulator of several key

\footnotetext{
*Correspondence: Dr C Montagut, Medical Oncology Department, Hospital del Mar, Passeig Marítim 25-29 08003 Barcelona, Spain;

E-mail: cmontagut@hospitaldelmar.cat

${ }^{7}$ These two authors contributed equally to this work.

Revised 4 February 2010; accepted 17 February 20I0; published online 16 March 2010
}

oncogenic pathways, together with the fact that EGFR is frequently expressed in colorectal cancer (CRC), has made it an excellent therapeutic target for the treatment of CRC patients.

The approval of two anti-EGFR monoclonal antibodies (moAbs), cetuximab and panitumumab, for metastatic CRC (mCRC) has dramatically improved the outcome of these patients. Both cetuximab and panitumumab have shown response rates (RRs) of $10-15 \%$ as single agents (Cunningham et al, 2004; Van Cutsem et al, 2007) and $\sim 20 \% \mathrm{RR}$ for cetuximab in combination with chemotherapy in unselected advanced mCRC patients (Cunningham et al, 2004). Moreover, anti-EGFR moAbs improve survival as salvage therapy in advanced mCRC (Jonker et al, 2007; Van Cutsem et al, 2007; Karapetis et al, 2008). The recent validation of KRAS mutations as a biomarker of negative response (or biomarker of resistance) to anti-EGFR therapies has meant a major revolution in the field of targeted therapies and in the treatment of mCRC patients (Amado et al, 2008; Karapetis et al, 2008; Bokemeyer et al, 2009; Van Cutsem et al, 2009). Activating mutations of the downstream EGFR protein KRAS are present in $\sim 40 \%$ of CRC patients. The most frequent KRAS mutations occur 
on codons 12 and 13, which impair ATPase activity, leading to a permanent activation of the KRAS-RAF-MEK-ERK pathway, despite EGFR blocking by cetuximab or panitumumab (Benvenuti et al, 2007; Di Fiore et al, 2007; De Roock et al, 2008; Lievre et al, 2008). After preliminary results in a retrospective cohort analysis (Lievre et al, 2008), the use of KRAS mutations as a marker of resistance to anti-EGFR moAbs was supported by a retrospective tumoural analysis of patients included in a randomised phase III clinical trial of panitumumab monotherapy $v s$ best supportive care in chemotherapy-refractory mCRC patients. KRAS mutant patients had a statistically significant lower RR (0\% vs 17\%) and shorter progression-free survival (Amado et al, 2008). In the case of cetuximab, results of a retrospective analysis of KRAS mutational status in tumour biopsy samples of patients included in several randomised phase III clinical trials have confirmed that KRAS is a solid biomarker of resistance to cetuximab. The CO.17 clinical trial showed that clinical benefit to salvage cetuximab monotherapy was confined to wild-type patients (Karapetis et al, 2008). In the OPUS and CRYSTAL trials, the addition of cetuximab to conventional chemotherapy in first-line treatment for mCRC patients showed no clinical benefit in KRAS mutated patients, whereas KRAS wild-type patients had $\sim 60 \%$ of clinical responses and longer progression-free survival with the addition of cetuximab (Bokemeyer et al, 2009; Van Cutsem et al, 2009). Therefore, the Food and Drug Administration has recently restricted the indication for panitumumab and cetuximab to wildtype KRAS mCRC patients. However, wild-type KRAS does not guarantee response to anti-EGFR moAb, and a fraction of KRAS wild-type patients will receive cetuximab or panitumumab without deriving any benefit. Thus, it is now crucial to find other markers of response that will help us in selecting KRAS wild-type patients most likely to respond - or not respond - to anti-EGFR therapy.

Although other mutationally activated protein kinases downstream of EGFR are being evaluated as potential biomarkers of resistance to cetuximab, so far none of them has sufficient supporting evidence to be routinely used in clinical practice. The V600E BRAF mutation is present in $\sim 5-10 \%$ of CRC patients and is mutually exclusive with KRAS mutations. In a hypothesisgenerating retrospective analysis of tumour biopsy samples of 113 patients treated with anti-EGFR moAb plus chemotherapy, BRAFmutated patients did not respond to therapy and had a shorter progession-free survival and overall survival (OS) compared with BRAF wild-type patients (Di Nicolantonio et al, 2008). Nevertheless, these results have not been confirmed when retrospectively analysing the BRAF mutational status of a large phase III clinical trial that evaluated chemotherapy, anti-angiogenic therapy and cetuximab in first-line treatment of mCRC patients (Tol et al, 2009). The role of BRAF mutations as a prognostic factor, as well as its low prevalence, will limit the confirmation of BRAF as a marker of resistance to anti-EGFR therapies (Di Nicolantonio et al, 2008; Tol et al, 2009; Roth et al, 2010). Activating mutations of PI3K are present in $\sim 10-30 \%$ of mCRC patients and have also been suggested to be a negative biomarker of response to cetuximab, although there are contradictory results from different studies (Ogino et al, 2009; Prenen et al, 2009; Sartore-Bianchi et al, 2009). Upregulation of the PI3K-Akt axis by loss of PTEN is theoretically another marker of resistance to anti-EGFR moAb. Despite several positive retrospective series, the studies are limited by a lack of standardised PTEN immunohistochemistry scoring (Frattini et al, 2007; Loupakis et al, 2009; Sartore-Bianchi et al, 2009). Upregulation of EGFR ligands - amphiregulin and epiregulin - expression has also been shown to be a marker of resistance in an expression-array analysis (Khambata-Ford et al, 2007), and has been confirmed in a large retrospective study (Jacobs et al, 2009). TP53 mutations are another potential mechanism of resistance to anti-EGFR under evaluation (OdenGangloff et al, 2009).
Mitogen-activated protein kinase phosphatases (MKPs), also known as dual-specificity phosphatases, regulate the activity of MAPKs (ERK, JNK and p38) by dephosphorylating both threonine and tyrosine residues and therefore deactivating them $(\mathrm{Wu}, 2007$; Keyse, 2008). The MKP family is composed of 12 members, of which MKP-1 (also referred to as dual-specificity phosphatase-1 and CL100) is the best characterised. MKP-1 is located in the nucleus and regulates the three MAPKs with different substrate preferences based on cell type and context (Chu et al, 1996; Keyse (2008)). MKP-1 is overexpressed in human tumours such as lung, breast and colon cancer, and has been involved in tumourigenesis (Loda et al, 1996; Vicent et al, 2004; Rojo et al, 2009). Oncogenic activation of MKP-1 is mediated by EGFR signalling by poorly understood mechanisms that include transcriptional regulation mediated by p38 and ERK (Xing et al, 1996; Li et al, 2001) and post-translational control by ERK (Brondello et al, 1999; Lin et al, 2003; Lin and Yang, 2006). Interestingly, our group and others are providing increasing evidence for a role of MKP-1 in acquisition of resistance to anti-cancer therapy. The molecular mechanism underlying MKP-1-mediated resistance to anti-cancer drugs is in part due to the activation of JNK-driven apoptosis by several anti-tumour agents such as anthracyclines, taxanes, cisplatin, proteasome inhibitors and more recently antiEGFR drugs. High levels of MKP-1 inhibit JNK and counterbalance the cytotoxic effects of such drugs (Sanchez-Perez et al, 1998, 2000; Small et al, 2004, 2007; Wang et al, 2006, 2007; Workman and de Bono, 2008; Rojo et al, 2009). With respect to anti-EGFR therapy, in vitro results with the anti-EGFR drug AG1478 showed that MKP1 -modulated JNK activation was critical for drug-induced apoptosis. Moreover, ectopic expression of MKP-1 suppressed JNK-mediated AG1417 apoptosis, leading to resistance to anti-EGFR therapy (Takeuchi et al, 2009). Thus, MKP-1 overexpression is a potential negative biomarker of response to certain anti-cancer agents including anti-EGFR therapy. Furthermore, preliminary pre-clinical work from our group found an association between high MKP-1 expression and resistance to cetuximab in CRC cell lines with wildtype KRAS. MKP-1 expression was markedly lower in DiFi cells (sensitive to cetuximab) compared with SW48 cells (resistant to cetuximab) (A Dalmases and C Montagut, unpublished data).

On the basis of the extensive evidence of the implication of MKP-1 in resistance to anti-cancer agents, as well as on the preliminary pre-clinical evidence by our group and others of the implication of MKP-1 in anti-EGFR therapy resistance (Takeuchi et al, 2009), we hypothesised that MKP-1 expression in human CRC tumours may be a marker of resistance to anti-EGFR moAb. We therefore analysed the impact of MKP-1 on clinical outcome in mCRC patients treated with cetuximab, with a special interest in KRAS wild-type patients.

\section{MATERIALS AND METHODS}

\section{Patient characteristics and clinical evaluation}

We retrospectively selected 48 consecutive patients with histologically confirmed mCRC treated with cetuximab-based chemotherapy at Hospital del Mar between 2004 and 2009. Patients' selection criteria were based on tumour tissue availability for molecular analysis. Cetuximab was administered as a loading dose of $400 \mathrm{mg} \mathrm{m}^{-2}$, followed by $250 \mathrm{mg} \mathrm{m}^{-2}$ every week intravenously. Clinical data and follow-up were obtained from the patients' medical records. Tumour response was evaluated retrospectively according to the response evaluation criteria in solid tumours (Therasse et al, 2000). Patients with complete response, partial response or stable disease were considered to have controlled disease (CD) (Oden-Gangloff et al, 2009). This study was approved by the Ethics Board of the Hospital and was performed according to Institutional Guidelines. 


\section{Mutational analyses}

DNA was extracted from two $15-\mu \mathrm{m}$ sections of paraffin-embedded tissue using a QIAamp Tissue Kit (QIAGEN GmbH, Hilden, Germany) according to the manufacturer's protocol. In those cases in which $<50 \%$ of tumoural cells were present in the sample, manual microdissection of tumoural tissue was performed. Primers for KRAS (codons 12 and 13) and BRAF V600E amplification were designed using Primer Express software (Applied Biosystems, Foster City, CA, USA) using NG_007524.1 (KRAS) and NG_007873.1 (BRAF) sequences and were as follows: KRAS-F: 5'-TTACGATACACGTCTGCAGTCAAC-3'; KRAS-R: 5' -AAAGAAT GGTCCTGCACCAGTAATA-3'; BRAF-F: $5^{\prime}$-CGGCTCCTAAAGCAA TGGC-3'; BRAF-R: $5^{\prime}$-CAGCATCTCAGGGCCAAAAA- $3^{\prime}$. DNA amplification was performed by PCR under the following conditions: initial denaturation for $10 \mathrm{~min}$ at $95^{\circ} \mathrm{C}, 40$ cycles consisting of: $1 \mathrm{~min}$ at $95^{\circ} \mathrm{C}, 1 \mathrm{~min}$ at $54^{\circ} \mathrm{C}$ (KRAS) or at $55^{\circ} \mathrm{C}(\mathrm{BRAF})$ and $1 \mathrm{~min}$ at $72{ }^{\circ} \mathrm{C}$, and a final step at $72{ }^{\circ} \mathrm{C}$ for $10 \mathrm{~min}$. Mutation analysis was performed by direct sequencing with BigDye v3.1 (Applied Biosystems) according to the manufacturer's instructions and analysed on an ABI3730XLSequencer (Applied Biosystems).

\section{Immunohistochemistry}

Immunohistochemistry was performed according to the methodology previously described by our group (Rojo et al, 2009). In brief, formalin-fixed paraffin-embedded $3 \mu \mathrm{m}$ tissue sections were used for immunostaining using the Dako-Link platform. After deparaffinisation in xylene and graded alcohols, heat antigen retrieval was carried out in pH9 EDTA-based buffer (Dako, Carpinteria, CA, USA). Endogenous peroxidase was blocked by immersing the sections in $0.03 \%$ hydrogen peroxide for $5 \mathrm{~min}$. Slides were incubated with anti-MKP-1 primary antibody for $1 \mathrm{~h}$ at room temperature, followed by incubation with the appropriate anti-Ig horseradish peroxidase-conjugated EnVision polymer (Dako) to detect antigen-antibody complexes. Sections were then visualised with $3,3^{\prime}$-diaminobezidine as chromogen and counterstained with haematoxylin. The specificity of the staining with anti-MKP-1 antibody was controlled by pre-incubating the antibody with antigen (blocking peptide), and performing immunostaining of tissue sections and immunoblotting of BT-474 breast cancer cell extracts. In addition to human specimens, renal tissues were obtained from wild-type and MKP-1 gene knockout mice, generously provided by Bristol-Myers Squibb Co. (Princeton, NJ, USA), and used for validation of MKP-1 assay. These mouse samples were processed with the same reagents and procedures as used for human samples.

Immunohistochemical evaluation was carried out by three independent observers (MI, MA and FR). To score a cell as positive, nuclear staining was required for MKP-1 expression. The expression was evaluated by calculating a semiquantitative histoscore (H-score) that included the determination of both the percentage of stained target cells and staining intensity (low, medium or high), as described in Rojo et al, (2009). The final score was determined after applying a weighting factor to each estimate and the following formula was used: $\mathrm{H}$-score $=($ low $\%) \times 1+($ medium $\%) \times 2+($ high $\%) \times 3$; the results ranged from 0 to 300 . MKP-1 was expressed in histologically normal colon epithelial cells and the pattern and intensity of staining were similar in all assayed specimens. MKP-1 overexpression was considered when the intensity of staining in the nuclei of tumour cells was higher than that observed in corresponding normal epithelial cells (Rojo et al, 2009).

\section{Statistical analysis}

Fisher's exact test was used to evaluate the association between MKP-1 expression with dichotomous clinical and molecular variables. Response to cetuximab-based therapy (responders vs non-responders) according to KRAS or BRAF mutational status or MKP-1 or EGFR expression was assessed by Fisher's exact test. The time to progression (TTP) was defined as the time from the start of cetuximab-based treatment until documented tumour progression or death. The Kaplan-Meier method was used to estimate TTP and OS and the log-rank test to compare survival curves. All statistical tests were conducted at the two-sided 0.05 level of significance. Statistical analysis was performed with SPSS Statistical Software, 17.0 version (SPSS, Inc., Chicago, IL, USA).

\section{RESULTS}

\section{Patient baseline characteristics and clinical response to cetuximab}

A total of 48 patients with mCRC treated with cetuximab-based chemotherapy were included in this study. Of them, 47 had been previously treated with chemotherapy, most of them $(83 \%)$ had previously received two or more lines of salvage treatment. Administration of cetuximab was combined with irinotecan in $92 \%$ of the patients. Evaluation of response to cetuximab based-therapy showed that 11 patients responded to treatment (11 partial responses; 0 complete responses) with a median TTP of 27 weeks (range 1-66 weeks). Non-responders (stable disease in 15 patients; progression disease in 22 patients) had a median TTP of 13 weeks (range 4-65 weeks). Patient baseline characteristics are shown on Table 1.

\section{KRAS mutational status and clinical response to cetuximab}

The mutational status of KRAS was assessed in all 48 patients included in the study. KRAS mutations were found in 12 patients $(25 \%)$. Such a low percentage compared with that in previous reports is probably due to the fact that after Health Authorities approval to limit the use of anti-EGFR moAb to KRAS wild-type mCRC patients, KRAS mutant cases have not received cetuximab-based therapy at our Institution. KRAS mutations were as follows: G13D in five patients; G12D in three patients; G12V in three patients; and G12A in one patient. In all, 11 out of 36 KRAS wild-type patients $(30 \%)$ responded to cetuximab, whereas none of the 12 patients $(0 \%)$ harbouring a KRAS mutation had a partial response $(P=0.04)$. We also measured $C D$, as recently reported (Oden-Gangloff et al, 2009). KRAS wild-type patients showed statistically significant improvement in CD compared with mutant KRAS patients $(69 \%$ vs $8 \%$, respectively). The median TTP for KRAS wild-type patients was 25 weeks $v s 8$ weeks for KRAS mutant patients $(P=0.01)$. Patients carrying KRAS-mutated tumours tended to have shorter OS, although the difference did not reach statistical significance $(P=0.1)$. Taken together, such findings confirm that KRAS mutations inversely correlate with clinical benefit from cetuximab therapy (Figure 1).

\section{BRAF mutational status and clinical response to cetuximab}

Three patients $(6 \%)$ harboured BRAF mutations, which were mutually exclusive with the presence of KRAS mutations. None of the three patients with BRAF mutations responded to cetuximab compared with $33 \%$ responses to cetuximab in BRAF wild-type patients. This difference was not statistically significant, probably because of the low incidence of BRAF $(P=0.54)$. Median TTP was higher in wild-type BRAF patients than in mutant BRAF patients (25 vs 7 weeks), although this correlation did not reach statistical significance $(P=0.53)$.

\section{MKP-1 expression and correlation with clinical and molecular characteristics}

Activated MKP-1 (i.e., nuclear staining), as assessed by immunohistochemistry, was overexpressed in 16 patients (33\%; Table 1$)$. 
Table I Patient baseline characteristics and clinical response by MKP-I status

\begin{tabular}{|c|c|c|}
\hline $\begin{array}{l}\text { Characteristics } \\
\text { (number of patients) }\end{array}$ & $\begin{array}{c}\text { Number (\%) } \\
\text { overexpressing MKP-I }\end{array}$ & $P$-value \\
\hline Number of patients $(n=48)$ & $16(33)$ & \\
\hline \multicolumn{3}{|l|}{ Age (years) } \\
\hline$<65(n=26)$ & $7(27)$ & \multirow[t]{2}{*}{ NS } \\
\hline$\geqslant 65(n=22)$ & $9(41)$ & \\
\hline \multicolumn{3}{|l|}{ Sex } \\
\hline Male $(n=31)$ & $12(39)$ & \multirow{2}{*}{ NS } \\
\hline Female $(n=17)$ & $4(23)$ & \\
\hline \multicolumn{3}{|l|}{ Site of primary tumour } \\
\hline Colon $(n=36)$ & II (30) & \multirow{2}{*}{ NS } \\
\hline Rectum $(n=12)$ & $5(42)$ & \\
\hline \multicolumn{3}{|l|}{ Tumour size } \\
\hline $\mathrm{TI}-\mathrm{T} 2(n=2)$ & $0(0)$ & \multirow[t]{2}{*}{ NS } \\
\hline $\mathrm{T} 3-\mathrm{T} 4(n=46)$ & $16(35)$ & \\
\hline \multicolumn{3}{|l|}{ Nodal status } \\
\hline Negative $(n=4)$ & $2(50)$ & \multirow[t]{2}{*}{ NS } \\
\hline Positive $(n=44)$ & $14(32)$ & \\
\hline \multicolumn{3}{|l|}{ Cetuximab regimen } \\
\hline Irinotecan based $(n=44)$ & $15(34)$ & \multirow[t]{2}{*}{ NS } \\
\hline Oxaliplatin based $(n=4)$ & I (25) & \\
\hline \multicolumn{3}{|l|}{ Number of previous chemotherapy } \\
\hline$<2(n=8)$ & $3(37)$ & \multirow[t]{2}{*}{ NS } \\
\hline$\geqslant 2(n=40)$ & $13(32)$ & \\
\hline \multicolumn{3}{|l|}{ Sites of metastasis } \\
\hline Hepatic $(n=33)$ & $13(39)$ & NS \\
\hline Lung $(n=21)$ & $7(33)$ & NS \\
\hline Peritoneal $(n=13)$ & $5(38)$ & NS \\
\hline Other $(n=16)$ & $4(25)$ & NS \\
\hline \multicolumn{3}{|l|}{ Metastasectomy } \\
\hline Yes $(n=6)$ & $3(50)$ & \multirow[t]{2}{*}{ NS } \\
\hline No $(n=42)$ & $13(31)$ & \\
\hline \multicolumn{3}{|l|}{ Mutational status } \\
\hline KRAS mutation $(n=12)$ & $3(25)$ & NS \\
\hline BRAF mutation $(n=3)$ & $3(100)$ & 0.04 \\
\hline \multicolumn{3}{|l|}{ Clinical response } \\
\hline Partial response $(n=11)$ & I (9) & \\
\hline Stable disease $(n=15)$ & $2(13)$ & \\
\hline Progression disease $(n=22)$ & $13(59)$ & \\
\hline
\end{tabular}

Abbreviations: MKP-I = mitogen-activated protein kinase phosphatase-I; NS = not significant.

Occasional and faint cytoplasmic MKP-1 was noted in malignant cells. Histologically normal epithelial cells exhibited weak and diffuse MKP-1 staining in the nuclei. A low level of MKP-1 expression was also present in stroma cells (fibroblast and endothelial cells) but it was not detected in lymphocytes (Figure 2).

No significant correlation was found between MKP-1 expression and clinical characteristics of patients, including age $(<65 v s \geqslant 65$ years old), sex, tumour primary site (colon $v s$ rectum), tumour size (T1-2 vs T3-4), nodal status (positive vs negative), cetuximab regimen (irinotecan $v s$ oxaliplatin), number of previously received chemotherapy metastatic lines $(<2 v s \geqslant 2$ lines), hepatic, lung, ascites and other metastases (present $v s$ absent for each metastatic site) and metastasectomy. MKP-1 expression was not linked to

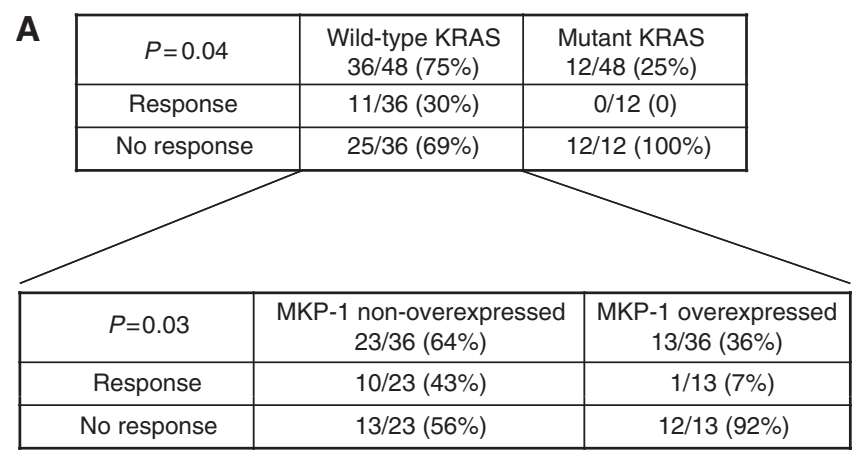

B
Wild-type KRAS

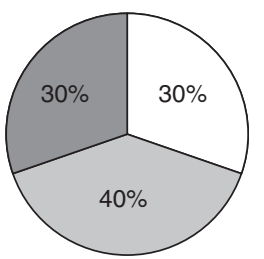

MKP-1 non-overexpressing

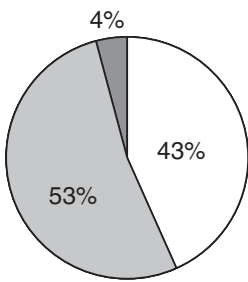

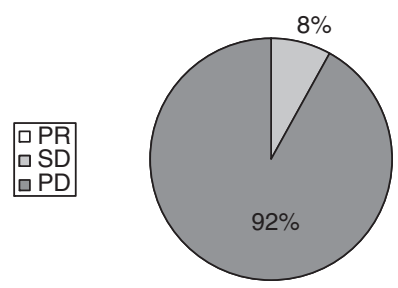

MKP-1 overexpressing

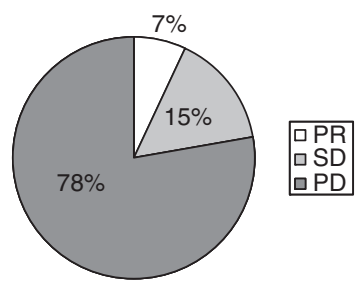

Figure I KRAS mutations correlate with a lack of response to cetuximab. In KRAS wild-type patients, mitogen-activated protein kinase phosphatase-I (MKP-I) overexpression is inversely correlated with response to cetuximab. (A) The number (and percentage) of patients with response and non-response (stable disease (SD) + progressive disease $(\mathrm{PD})$ ) to cetuximab are indicated according to KRAS mutational status and MKP-I expression. (B) Pie charts showing the percentage of patients showing partial response (PR), SD and PD according to KRAS mutational status and MKP-I expression.

expression of EGFR as assessed by immunohistochemistry $(P=0.61)$. The expression of MKP-1 was not correlated with KRAS mutational status as shown by $19 \%$ of MKP-1 overexpressors harbouring a KRAS mutation $v s 28 \%$ of MKP-1 nonoverexpressors with mutant KRAS $(P=0.72)$. It is worth noting that all three patients with BRAF mutations had MKP-1 overexpression $(P=0.04)$ (Table 1$)$.

\section{MKP-1 expression and clinical response to cetuximab-based treatment in all patients}

Only 1 of 16 patients with MKP-1 overexpression (6\%) had a partial response to treatment, whereas 10 of 32 non-overexpressing MKP-1 patients (31\%) responded to cetuximab, although the correlation was not statistically significant $(P=0.074)$. Moreover, CD was achieved in $19 \%$ of patients with MKP-1 overexpression, compared with $72 \%$ of patients with low MKP-1 protein levels $(P=0.001)$. The median TTP was lower in MKP-1 overexpressing patients, compared with patients with non-overexpressed MKP-1 (13 vs 27 weeks; $P=0.43$ ). No statistically significant differences were observed by OS analysis $(P=0.5)$. 
A

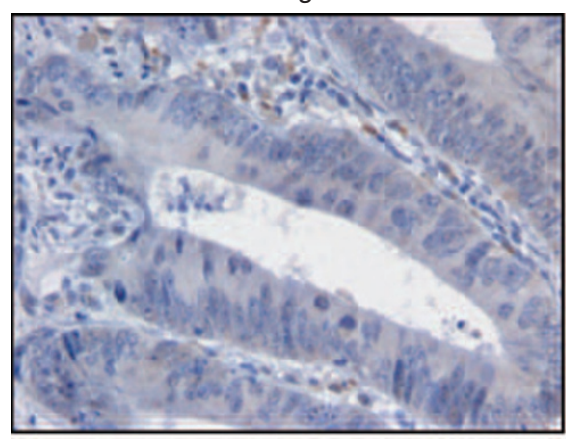

MKP-1 intense expression

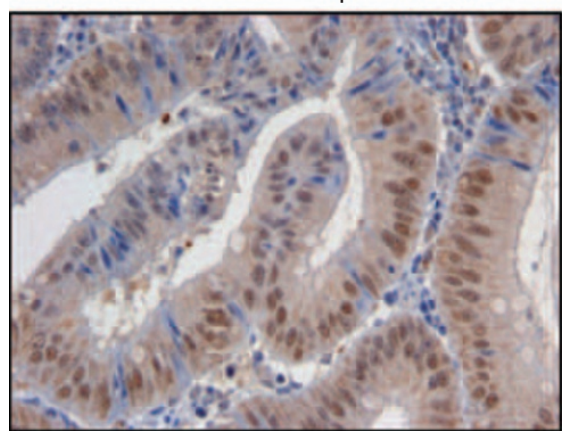

MKP-1 low expression

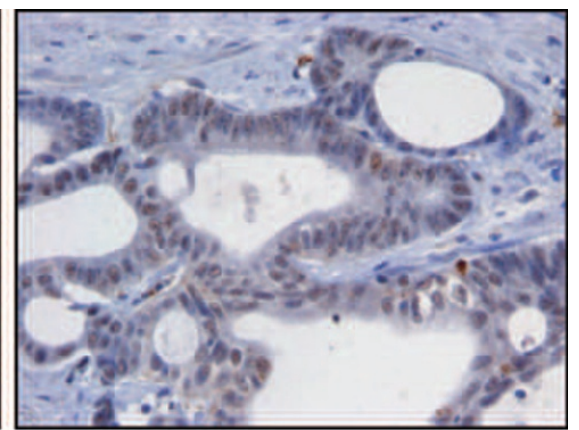

MKP-1 intense expression

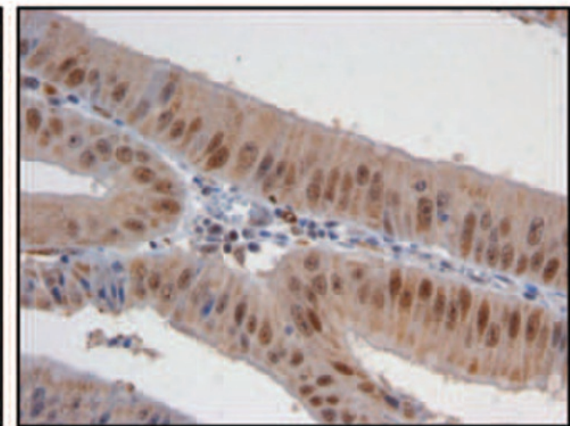

$\mathrm{KO}-\mathrm{MKP}-1$
C

\section{Positive control}

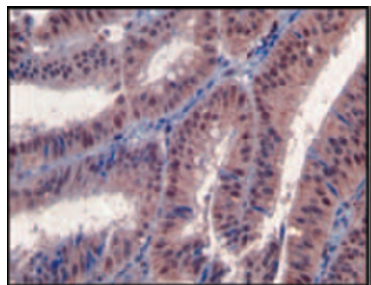

Negative control sc-1199P pre-incubation

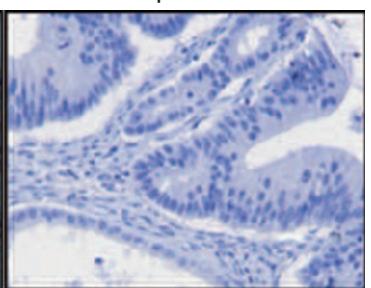

D

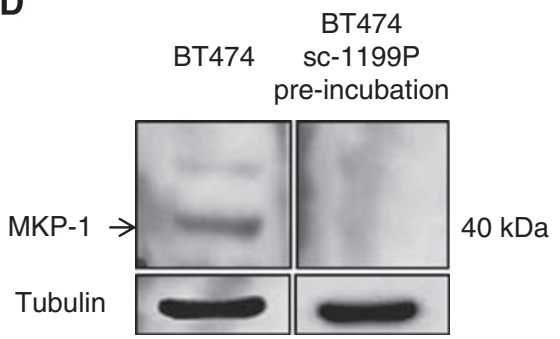

Figure 2 Mitogen-activated protein kinase phosphatase-I (MKP-I) expression in colon cancer. (A) Representative colon adenocarcinoma specimens showing undetected (negative) MKP-I expression, low expression and intense diffuse staining in tumour cells. Malignant cells expressed MKP-I nuclear staining; mild cytoplasmic staining is occasionally noted. Stroma cells (fibroblast and endothelial cells) also showed a low level of MKP-I expression, which is not detected in lymphocytes. (B) The specificity of immunostaining was probed by assaying renal tissue sections obtained from wild-type and MKP-I gene knockout mice. Specimens were processed using the same reagents and procedures as used in human samples. No staining was observed in knockout tissue and intense nuclear expression was detected in renal tubes. (C) Positive and negative controls. The same tumour specimen considered as positive control was assayed by pre-incubation of primary antibody with a specific blocking peptide, showing no staining. (D) MKP-I expression explored by western blot from BT-474 total lysates, using the same primary antibody and pre-incubated with a blocking peptide. The molecular size of MKP-I was $\sim 40 \mathrm{kD}$. The protein was not detected under blocking peptide pre-incubation.

\section{MKP-1 expression and clinical response to cetuximab in KRAS wild-type patients}

To address the clinically relevant need to identify KRAS wild-type patients who may respond to cetuximab, we analysed MKP-1 expression in the subset of KRAS wild-type patients. Among KRAS wild-type patients, only 1 out of 13 MKP-1 overexpressing patients
(7\%) responded to cetuximab, whereas 10 out of 23 patients $(43 \%)$ with non-overexpressed MKP-1 responded to cetuximab $(P=0.03$, Figure 1). Furthermore, wild-type KRAS patients with low MKP-1 levels had $96 \% \mathrm{CD}$ as compared with $22 \% \mathrm{CD}$ in wild-type KRAS patients with high MKP-1 levels $(P<0.0001$, Figure 1$)$. Patients with wild-type KRAS and overexpressed MKP-1 had a shorter median TTP than wild-type KRAS patients with 


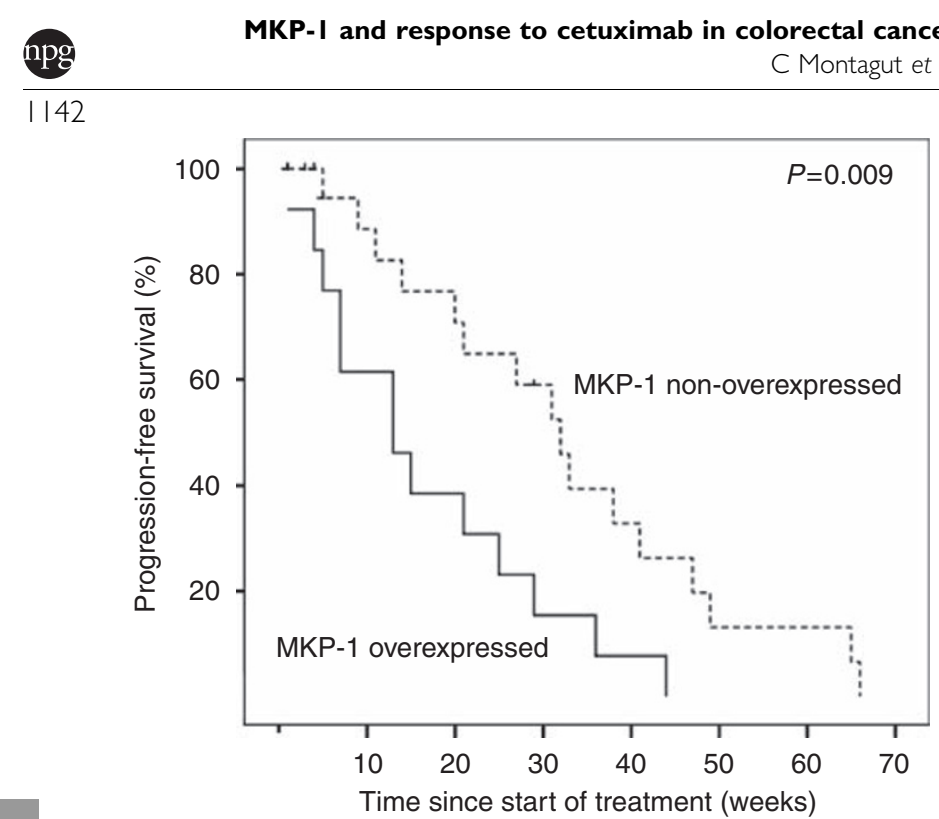

Figure 3 Patients with mitogen-activated protein kinase phosphatase(MKP-I) overexpression show shorter median time to progression (TTP) than MKP-I non-overexpressing patients in KRAS wild-type colon cancer patients treated with cetuximab ( 13 vs 32 weeks, $P=0.009$ ).

non-overexpressed MKP-1 (13 vs 32 weeks, $P=0.009$, Figure 3). KRAS wild-type individuals with non-overexpressed MKP-1 showed a trend towards longer OS, which was not statistically significant $(P=0.1)$. Such results suggest MKP-1 as a potential predictive factor of failure to respond to cetuximab in KRAS wild-type patients.

\section{MKP-1 expression and clinical response to cetuximab in KRAS and BRAF wild-type patients}

Given the increasing amount of evidence on a role for BRAF as a marker of resistance to anti-EGFR moAb and to exclude a possible bias, the predictive role of MKP-1 to cetuximab-based therapy was assessed in KRAS and BRAF wild-type patients. Among KRAS/ BRAF wild-type patients, only 1 out of 10 patients (10\%) with overexpressed MKP-1 responded to cetuximab, compared with 10 out of 23 patients $(43 \%)$ with non-overexpressed MKP-1 $(P=0.1)$. It is of importance that $\mathrm{CD}$ was only observed in two patients with high MKP-1 expression levels, whereas 22 of the 23 patients with MKP-1 low expression had CD $(P=0.0001)$. Among KRAS and BRAF wild-type patients, those with MKP-1 overexpression had shorter TTP than patients with low MKP-1 levels (32 vs 13 weeks, $P=0.006)$.

\section{DISCUSSION}

This study suggests MKP-1 as a novel and promising biomarker of response to cetuximab in mCRC patients, particularly relevant to certain KRAS wild-type patients who will not benefit from cetuximab therapy.

The selection of patients to be treated with targeted therapies based on useful and validated biomarkers is crucial to maximise clinical efficacy while minimising toxicities and optimising the use of currently constrained financial resources (Baselga, 2006; McDermott et al, 2007; Workman and de Bono, 2008). The validation of KRAS mutations as a negative biomarker of response to anti-EGFR therapies such as cetuximab has meant a major revolution in the treatment of CRC patients. The number of KRAS mutations in this study (25\%) was lower than that previously reported, partly because of the fact that after June 2008, mutant KRAS patients were not treated with cetuximab at our Institution according to the results presented at the 44th ASCO Annual
Meeting (Bokemeyer et al, 2009; Van Cutsem et al, 2009). As expected, in our series, clinical response was confined to wild-type KRAS patients, who also had a statistically significantly longer TTP than mutant KRAS patients.

In this study, BRAF mutations correlated with resistance to cetuximab-based therapy, although the decrease in median TTP was not statistically significant. We cannot rule out that this may be related to an insufficient sample size to detect differences in such a low-frequency occurring event. The response data confirm the results of previous studies that showed that BRAF mutations were linked to cetuximab efficacy in mCRC patients (Di Nicolantonio et al, 2008). Nevertheless, recent results in mCRC patients treated with chemotherapy, bevacizumab and cetuximab as first-line therapy showed that BRAF mutations had a role as a prognostic factor but not as a predictive marker of response to cetuximab (Tol et al, 2009). Moreover, BRAF mutations have prognostic value in stage II and III CRC patients (Roth et al, 2010). Such apparently contradictory results may in part be explained by the possible acquisition of BRAF mutations in advanced stages of the disease and suggest that the role of BRAF as a predictive marker of resistance to cetuximab might be restricted to patients previously treated with chemotherapy. In this sudy, $83 \%$ of patients had received two or more previous lines of chemotherapy, and only one patient was treated with cetuximab as first-line therapy for mCRC. Another controversial marker of anti-EGFR moAb efficacy is the target itself. EGFR protein, as assessed by immunohistochemistry, was overexpressed in $76 \%$ of tumours (data not shown) and did not correlate with response to cetuximab as previously demonstrated in other studies (Cunningham et al, 2004; Saltz et al, 2004; Chung et al, 2005).

Our data showed that overexpression of MKP-1, as assessed by immunohistochemistry, was correlated with resistance to cetuximab-based chemotherapy in mCRC patients, and suggests a role for MKP-1 as a negative predictive biomarker of response to cetuximab, particularly in KRAS wild-type patients. Although this study is the first to correlate MKP-1 levels to cetuximab resistance in CRC patients, the implication of other MKPs in modulating response to anti-EGFR therapy has previously been suggested. A gene expression profile analysis identified high MKP-2 levels (also known as dual-specificity phosphatase-4) in cetuximab-resistant patients (Khambata-Ford et al, 2007). Moreover, an elegant abstract presented at the 2009 ASCO Annual Meeting showed that MKP-2 expression levels as determined by quantitative reverse transcriptase PCR identifies a subgroup of patients with shorter median OS to cetuximab therapy among KRAS wild-type mCRC patients (De Roock et al, 2009).

As all patients included in this study received cetuximab in combination with chemotherapy (irinotecan in $92 \%$ of the patients), it cannot be excluded that MKP-1-based clinical outcome is influenced, at least in part, by its interaction with chemotherapy. Although so far there is no reported evidence of a role of MKP-1 in conferring resistance to irinotecan, it will certainly be necessary to conduct randomised clinical trials and extensive pre-clinical modelling to exclude such hypotheses. A recently published paper reports that other cetuximab predictive markers such as KRAS and BRAF mutations do not preclude benefit from irinotecan or oxaliplatin chemotherapy (Richman et al, 2009).

Molecular mechanisms underlying MKP-1-mediated resistance to cetuximab are poorly understood. A possible explanation is inhibition of JNK-mediated apoptosis by high MKP-1 levels, as has recently been reported by Takeuchi et al (2009). On the other hand, a recently published interesting hypothesis-generating study supports p53 mutations as a potential marker of response to cetuximab (Oden-Gangloff et al, 2009). MKP-1 has been shown to be transcriptionally regulated by $\mathrm{p} 53$, and mutations of $\mathrm{p} 53$ abrogate $\mathrm{p} 53$-dependent transcription of MKP-1 in vitro (Yang and $\mathrm{Wu}, 2004$; Liu et al, 2008). Thus, it could be speculated that the association between p53 mutations and better clinical outcome in 
cetuximab-treated patients is in part explained by a decrease in the expression of MKP-1, although this molecular association needs to be further characterised.

Interestingly, mutant KRAS tumours have been shown to express high constitutive levels of MKP-1, MKP-2 and MKP-3, probably as part of the regulatory feedback loop to attenuate the high activation of ERK by mutant KRAS (Bild et al, 2006). Moreover, functional studies in a KRAS mutant CRC murine model has confirmed MKP-3 high levels, and high MKP-2 and MKP-3 expressions have been described in human tumour biopsy samples from mutant KRAS CRC patients (Haigis et al, 2008; De Roock et al, 2009). However, in this study, we found that MKP-1 basal levels were not linked to KRAS mutations. It is worth noting that the presence of BRAF V600E mutations was associated with MKP-1 overexpression in all the cases, although the number of patients was insufficient to achieve a significant correlation.

\section{REFERENCES}

Albanell J, Codony-Servat J, Rojo F, Del Campo JM, Sauleda S, Anido J, Raspall G, Giralt J, Rosello J, Nicholson RI, Mendelsohn J, Baselga J (2001) Activated extracellular signal-regulated kinases: association with epidermal growth factor receptor/transforming growth factor alpha expression in head and neck squamous carcinoma and inhibition by anti-epidermal growth factor receptor treatments. Cancer Res 61: $6500-6510$

Amado RG, Wolf M, Peeters M, Van Cutsem E, Siena S, Freeman DJ, Juan T, Sikorski R, Suggs S, Radinsky R, Patterson SD, Chang DD (2008) Wild-type KRAS is required for panitumumab efficacy in patients with metastatic colorectal cancer. J Clin Oncol 26: 1626-1634

Baselga J (2006) Targeting tyrosine kinases in cancer: the second wave. Science 312: $1175-1178$

Benvenuti S, Sartore-Bianchi A, Di Nicolantonio F, Zanon C, Moroni M, Veronese S, Siena S, Bardelli A (2007) Oncogenic activation of the RAS/ RAF signaling pathway impairs the response of metastatic colorectal cancers to anti-epidermal growth factor receptor antibody therapies. Cancer Res 67: 2643-2648

Bild AH, Yao G, Chang JT, Wang Q, Potti A, Chasse D, Joshi MB, Harpole D, Lancaster JM, Berchuck A, Olson Jr JA, Marks JR, Dressman HK, West M, Nevins JR (2006) Oncogenic pathways signatures in human cancers as a guide to targeted therapies. Nature 439: $353-357$

Bokemeyer C, Bondarenko I, Makhson A, Hartmann JT, Aparicio J, de Braud F, Donea S, Ludwig H, Schuch G, Stroh C, Loos AH, Zubel A, Koralewski P (2009) Fluorouracil, leucovorin, and oxaliplatin with and without cetuximab in the first-line treatment of metastatic colorectal cancer. I Clin Oncol 27: 663-671

Brondello JM, Pouyssegur J, McKenzie FR (1999) Reduced MAP kinase phosphatase-1 degradation after p42/p44MAPK-dependent phosphorylation. Science 286: 2514-2517

Cunningham D, Humblet Y, Siena S, Khayat D, Bleiberg H, Santoro A, Bets D, Mueser M, Harstrick A, Verslype C, Chau I, Van Cutsem E (2004) Cetuximab monotherapy and cetuximab plus irinotecan in irinotecan-refractory metastatic colorectal cancer. $N$ Engl J Med 351: $337-345$

Chu Y, Solski PA, Khosravi-Far R, Der CJ, Kelly K (1996) The mitogenactivated protein kinase phosphatases PAC1, MKP-1, and MKP-2 have unique substrate specificities and reduced activity in vivo toward the ERK2 sevenmaker mutation. J Biol Chem 271: 6497-6501

Chung KY, Shia J, Kemeny NE, Shah M, Schwartz GK, Tse A, Hamilton A, Pan D, Schrag D, Schwartz L, Klimstra DS, Fridman D, Kelsen DP, Saltz LB (2005) Cetuximab shows activity in colorectal cancer patients with tumors that do not express the epidermal growth factor receptor by immunohistochemistry. J Clin Oncol 23: 1803-1810

De Roock W, Jansenss M, Biesmans B, Jacobs J, De Schutter J, Fieuws E, Van Cutsem E, Tejpar S (2009) DUSPs as marker of MEK/Erk activation in primary colorectal cancer. J Clin Oncol 27: 15s. (abstr 4064)

De Roock W, Piessevaux H, De Schutter J, Janssens M, De Hertogh G, Personeni N, Biesmans B, Van Laethem JL, Peeters M, Humblet Y, Van Cutsem E, Tejpar S (2008) KRAS wild-type state predicts survival and is associated to early radiological response in metastatic colorectal cancer treated with cetuximab. Ann Oncol 19: 508-515
Collectively, our results suggest a role for MKP-1 in predicting failure to respond to cetuximab-based chemotherapy in KRAS wild-type CRC patients.

\section{ACKNOWLEDGEMENTS}

This work was supported by PI061513, PS0901491, PS0901285 and PJ091296 (Spanish Health Ministry Grant 'Fondo de Investigación Sanitaria'), RTICC 06/0020/19 and grant from DIUE (generalitat de Catalunya, 2009 SGR 321). We thank Fundació Cellex (Barcelona) for a generous donation to the Group of Molecular Therapeutics and Biomarkers, IMIM-Hospital del Mar. We thank the Tumour Bank of the Department of Pathology of Hospital del Mar and the Xarxa de Bancs de Tumors de Catalunya for providing tissue samples.

Dhillon AS, Hagan S, Rath O, Kolch W (2007) MAP kinase signalling pathways in cancer. Oncogene 26: $3279-3290$

Di Fiore F, Blanchard F, Charbonnier F, Le Pessot F, Lamy A, Galais MP, Bastit L, Killian A, Sesboue R, Tuech JJ, Queuniet AM, Paillot B, Sabourin JC, Michot F, Michel P, Frebourg T (2007) Clinical relevance of KRAS mutation detection in metastatic colorectal cancer treated by cetuximab plus chemotherapy. Br J Cancer 96: 1166-1169

Di Nicolantonio F, Martini M, Molinari F, Sartore-Bianchi A, Arena S, Saletti P, De Dosso S, Mazzucchelli L, Frattini M, Siena S, Bardelli A (2008) Wild-type BRAF is required for response to panitumumab or cetuximab in metastatic colorectal cancer. J Clin Oncol 26: 5705-5712

Frattini M, Saletti P, Romagnani E, Martin V, Molinari F, Ghisletta M, Camponovo A, Etienne LL, Cavalli F, Mazzucchelli L (2007) PTEN loss of expression predicts cetuximab efficacy in metastatic colorectal cancer patients. Br J Cancer 97: 1139-1145

Haigis KM, Kendall KR, Wang Y, Cheung A, Haigis MC, Glickman JN, Niwa-Kawakita M, Sweet-Cordero A, Sebolt-Leopold J, Shannon KM, Settleman J, Giovannini M, Jacks T (2008) Differential effects of oncogenic K-Ras and N-Ras on proliferation, differentiation and tumor progression in the colon. Nat Genet 40: 600-608

Hanahan D, Weinberg RA (2000) The hallmarks of cancer. Cell 100: 57-70

Jacobs B, De Roock W, Piessevaux H, Van Oirbeek R, Biesmans B, De Schutter J, Fieuws S, Vandesompele J, Peeters M, Van Laethem JL, Humblet Y, Penault-Llorca F, De Hertogh G, Laurent-Puig P, Van Cutsem E, Tejpar S (2009) Amphiregulin and epiregulin mRNA expression in primary tumors predicts outcome in metastatic colorectal cancer treated with cetuximab. J Clin Oncol 27: 5068-5074

Jonker DJ, O'Callaghan CJ, Karapetis CS, Zalcberg JR, Tu D, Au HJ, Berry SR, Krahn M, Price T, Simes RJ, Tebbutt NC, van Hazel G, Wierzbicki R, Langer C, Moore MJ (2007) Cetuximab for the treatment of colorectal cancer. N Engl J Med 357: 2040-2048

Karapetis CS, Khambata-Ford S, Jonker DJ, O'Callaghan CJ, Tu D, Tebbutt NC, Simes RJ, Chalchal H, Shapiro JD, Robitaille S, Price TJ, Shepherd L, Au HJ, Langer C, Moore MJ, Zalcberg JR (2008) K-ras mutations and benefit from cetuximab in advanced colorectal cancer. N Engl J Med 359: 1757-1765

Keyse SM (2008) Dual-specificity MAP kinase phosphatases (MKPs) and cancer. Cancer Metastasis Rev 27: 253-261

Khambata-Ford S, Garrett CR, Meropol NJ, Basik M, Harbison CT, Wu S, Wong TW, Huang X, Takimoto CH, Godwin AK, Tan BR, Krishnamurthi SS, Burris 3rd HA, Poplin EA, Hidalgo M, Baselga J, Clark EA, Mauro DJ (2007) Expression of epiregulin and amphiregulin and K-ras mutation status predict disease control in metastatic colorectal cancer patients treated with cetuximab. J Clin Oncol 25: 3230-3237

Li J, Gorospe M, Hutter D, Barnes J, Keyse SM, Liu Y (2001) Transcriptional induction of MKP-1 in response to stress is associated with histone $\mathrm{H} 3$ phosphorylation-acetylation. Mol Cell Biol 21: 8213-8224

Lievre A, Bachet JB, Boige V, Cayre A, Le Corre D, Buc E, Ychou M, Bouche O, Landi B, Louvet C, Andre T, Bibeau F, Diebold MD, Rougier P, Ducreux M, Tomasic G, Emile JF, Penault-Llorca F, Laurent-Puig P (2008) KRAS mutations as an independent prognostic factor in patients with advanced colorectal cancer treated with cetuximab. J Clin Oncol 26: 374-379 
Lin YW, Chuang SM, Yang JL (2003) ERK1/2 achieves sustained activation by stimulating MAPK phosphatase-1 degradation via the ubiquitinproteasome pathway. J Biol Chem 278: 21534-21541

Lin YW, Yang JL (2006) Cooperation of ERK and SCFSkp2 for MKP-1 destruction provides a positive feedback regulation of proliferating signaling. J Biol Chem 281: 915-926

Liu YX, Wang J, Guo J, Wu J, Lieberman HB, Yin Y (2008) DUSP1 is controlled by p53 during the cellular response to oxidative stress. $\mathrm{Mol}$ Cancer Res 6: 624-633

Loda M, Capodieci P, Mishra R, Yao H, Corless C, Grigioni W, Wang Y, Magi-Galluzzi C, Stork PJ (1996) Expression of mitogen-activated protein kinase phosphatase-1 in the early phases of human epithelial carcinogenesis. Am J Pathol 149: $1553-1564$

Loupakis F, Pollina L, Stasi I, Ruzzo A, Scartozzi M, Santini D, Masi G, Graziano F, Cremolini C, Rulli E, Canestrari E, Funel N, Schiavon G, Petrini I, Magnani M, Tonini G, Campani D, Floriani I, Cascinu S, Falcone A (2009) PTEN expression and KRAS mutations on primary tumors and metastases in the prediction of benefit from cetuximab plus irinotecan for patients with metastatic colorectal cancer. J Clin Oncol 27: $2622-2629$

McDermott U, Sharma SV, Dowell L, Greninger P, Montagut C, Lamb J, Archibald H, Raudales R, Tam A, Lee D, Rothenberg SM, Supko J, Sordella R, Ulkus LE, Maheswaran S, Njauw CN, Tsao H, Drew L, Hanke JH, Xiao-Jun M, Erlander MG, Gray N, Haber DA, Settleman J (2007) Identification of genotype-correlated sensitivity to selective kinase inhibitors using high-throughput tumor cell line profiling. Proc Natl Acad Sci USA 104: 19936-19941

Mendelsohn J, Baselga J (2006) Epidermal growth factor receptor targeting in cancer. Semin Oncol 33: 369-385

Oden-Gangloff A, Di Fiore F, Bibeau F, Lamy A, Bougeard G, Charbonnier F, Blanchard F, Tougeron D, Ychou M, Boissiere F, Le Pessot F, Sabourin JC, Tuech JJ, Michel P, Frebourg T (2009) TP53 mutations predict disease control in metastatic colorectal cancer treated with cetuximab-based chemotherapy. Br J Cancer 100: 1330-1335

Ogino S, Nosho K, Kirkner GJ, Shima K, Irahara N, Kure S, Chan AT, Engelman JA, Kraft P, Cantley LC, Giovannucci EL, Fuchs CS (2009) PIK3CA mutation is associated with poor prognosis among patients with curatively resected colon cancer. J Clin Oncol 27: 1477-1484

Prenen H, De Schutter J, Jacobs B, De Roock W, Biesmans B, Claes B, Lambrechts D, Van Cutsem E, Tejpar S (2009) PIK3CA mutations are not a major determinant of resistance to the epidermal growth factor receptor inhibitor cetuximab in metastatic colorectal cancer. Clin Cancer Res 15: $3184-3188$

Richman SD, Seymour MT, Chambers P, Elliot F, Daly CL, Meade AM, Taylor G, Barrett JH, Quirke P (2009) KRAS and BRAF mutations in advanced colorectal cancer are associated with poor prognosis but do not preclude benefit from oxaliplatin or irinotecan: results from the MRC FOCUS trial. J Clin Oncol 27: 5931 - 5937

Rojo F, Gonzalez-Navarrete I, Bragado R, Dalmases A, Menendez S, CortesSempere M, Suarez C, Oliva C, Servitja S, Rodriguez-Fanjul V, SanchezPerez I, Campas C, Corominas JM, Tusquets I, Bellosillo B, Serrano S, Perona R, Rovira A, Albanell J (2009) Mitogen-activated protein kinase phosphatase- 1 in human breast cancer independently predicts prognosis and is repressed by doxorubicin. Clin Cancer Res 15: 3530-3539

Roth AD, Tejpar S, Delorenzi M, Yan P, Fiocca R, Klingbiel D, Dietrich D, Biesmans B, Bodoky G, Barone C, Aranda E, Nordlinger B, Cisar L, Labianca R, Cunningham D, Van Cutsem E, Bosman F (2010) Prognostic role of KRAS and BRAF in stage II and III resected colon cancer: results of the translational study on the PETACC-3, EORTC 40993, SAKK 60-00 trial. J Clin Oncol 28: 466-474

Saltz LB, Meropol NJ, Loehrer Sr PJ, Needle MN, Kopit J, Mayer RJ (2004) Phase II trial of cetuximab in patients with refractory colorectal cancer that expresses the epidermal growth factor receptor. J Clin Oncol 22: $1201-1208$
Sanchez-Perez I, Martinez-Gomariz M, Williams D, Keyse SM, Perona R (2000) CL100/MKP-1 modulates JNK activation and apoptosis in response to cisplatin. Oncogene 19: $5142-5152$

Sanchez-Perez I, Murguia JR, Perona R (1998) Cisplatin induces a persistent activation of JNK that is related to cell death. Oncogene 16: $533-540$

Sartore-Bianchi A, Martini M, Molinari F, Veronese S, Nichelatti M, Artale S, Di Nicolantonio F, Saletti P, De Dosso S, Mazzucchelli L, Frattini M, Siena S, Bardelli A (2009) PIK3CA mutations in colorectal cancer are associated with clinical resistance to EGFR-targeted monoclonal antibodies. Cancer Res 69: $1851-1857$

Small GW, Shi YY, Edmund NA, Somasundaram S, Moore DT, Orlowski RZ (2004) Evidence that mitogen-activated protein kinase phosphatase-1 induction by proteasome inhibitors plays an antiapoptotic role. Mol Pharmacol 66: 1478 - 1490

Small GW, Shi YY, Higgins LS, Orlowski RZ (2007) Mitogen-activated protein kinase phosphatase-1 is a mediator of breast cancer chemoresistance. Cancer Res 67: 4459-4466

Takeuchi K, Ito F (2010) EGF receptor in relation to tumor development: molecular basis of responsiveness of cancer cells to EGFR-targeting tyrosine kinase inhibitors. Febs J 277: 316-326

Takeuchi K, Shin-ya T, Nishio K, Ito F (2009) Mitogen-activated protein kinase phosphatase- 1 modulated JNK activation is critical for apoptosis induced by inhibitor of epidermal growth factor receptor-tyrosine kinase. FEBS J 276: 1255 - 1265

Therasse P, Arbuck SG, Eisenhauer EA, Wanders J, Kaplan RS, Rubinstein L, Verweij J, Van Glabbeke M, van Oosterom AT, Christian MC, Gwyther SG (2000) New guidelines to evaluate the response to treatment in solid tumors. European Organization for Research and Treatment of Cancer, National Cancer Institute of the United States, National Cancer Institute of Canada. J Natl Cancer Inst 92: 205-216

Tol J, Nagtegaal ID, Punt CJ (2009) BRAF mutation in metastatic colorectal cancer. $N$ Engl J Med 361: $98-99$

Van Cutsem E, Kohne CH, Hitre E, Zaluski J, Chang Chien CR, Makhson A D'Haens G, Pinter T, Lim R, Bodoky G, Roh JK, Folprecht G, Ruff P, Stroh C, Tejpar S, Schlichting M, Nippgen J, Rougier P (2009) Cetuximab and chemotherapy as initial treatment for metastatic colorectal cancer. $N$ Engl J Med 360: $1408-1417$

Van Cutsem E, Peeters M, Siena S, Humblet Y, Hendlisz A, Neyns B, Canon JL, Van Laethem JL, Maurel J, Richardson G, Wolf M, Amado RG (2007) Open-label phase III trial of panitumumab plus best supportive care compared with best supportive care alone in patients with chemotherapyrefractory metastatic colorectal cancer. J Clin Oncol 25: 1658-1664

Vicent S, Garayoa M, Lopez-Picazo JM, Lozano MD, Toledo G, Thunnissen FB, Manzano RG, Montuenga LM (2004) Mitogen-activated protein kinase phosphatase-1 is overexpressed in non-small cell lung cancer and is an independent predictor of outcome in patients. Clin Cancer Res 10: $3639-3649$

Wang J, Zhou JY, Wu GS (2007) ERK-dependent MKP-1-mediated cisplatin resistance in human ovarian cancer cells. Cancer Res 67: 11933-11941

Wang Z, Xu J, Zhou JY, Liu Y, Wu GS (2006) Mitogen-activated protein kinase phosphatase-1 is required for cisplatin resistance. Cancer Res 66: $8870-8877$

Workman P, de Bono J (2008) Targeted therapeutics for cancer treatment: major progress towards personalised molecular medicine. Curr Opin Pharmacol 8: $359-362$

Wu GS (2007) Role of mitogen-activated protein kinase phosphatases (MKPs) in cancer. Cancer Metastasis Rev 26: 579-585

Xing J, Ginty DD, Greenberg ME (1996) Coupling of the RAS-MAPK pathway to gene activation by RSK2, a growth factor-regulated CREB kinase. Science 273: $959-963$

Yang H, Wu GS (2004) p53 Transactivates the phosphatase MKP1 through both intronic and exonic p53 responsive elements. Cancer Biol Ther 3: $1277-1282$ 